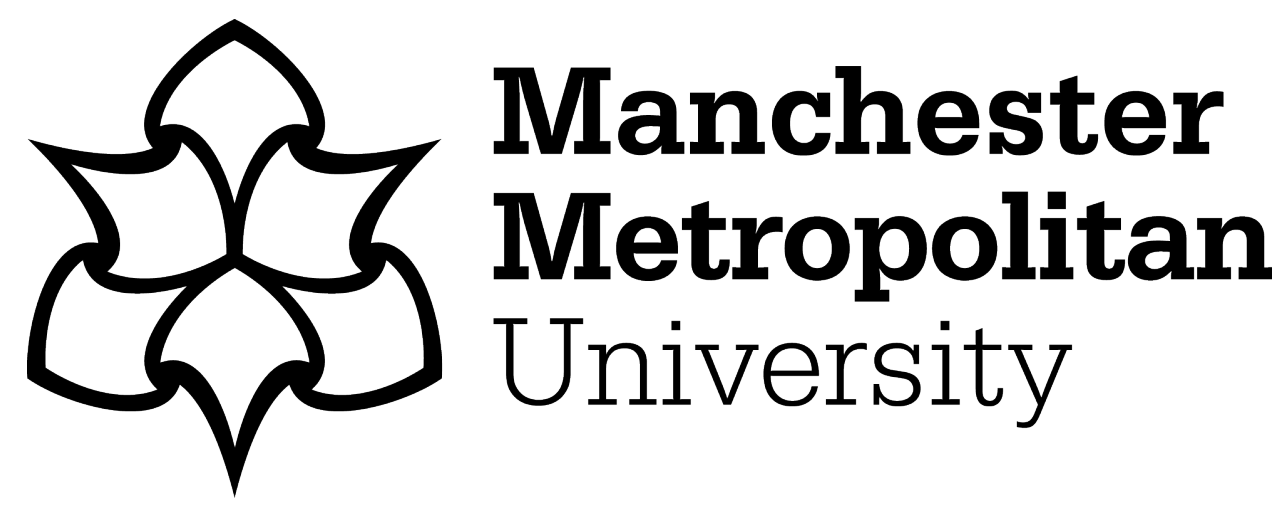

Farhan, L, Alissa, AE, Shukur, ST, Hammoudeh, M and Kharel, R (2018) An energy efficient long hop ( $\mathrm{LH}$ ) first scheduling algorithm for scalable Internet of Things (IOT) networks. In: Eleventh International Conference on Sensing Technology (ICST 2017), 04 December 2017 - 06 December 2017, Sydney, Australia.

Downloaded from: https://e-space.mmu.ac.uk/620829/

Publisher: IEEE

DOI: https://doi.org/10.1109/ICSensT.2017.8304511

Please cite the published version 


\section{An Energy Efficient Long Hop (LH) First Scheduling Algorithm for Scalable Internet of Things (IoT) Networks}

\author{
Laith Farhan \\ School of Engineering \\ Manchester Metropolitan University, UK \\ \& University of Diyala, Iraq \\ 1.al-bayati@mmu.ac.uk \\ Mohammad Hammoudeh \\ School of CMDT \\ Manchester Metropolitan University, UK \\ m.hammoudeh@mmu.ac.uk
}

\author{
Ali E. Alissa \\ School of Computer Science \\ University of Plymouth, UK \\ ali.alissa@plymouth.ac.uk
}

\author{
Sinan T. Shukur \\ Faculty of Medicine and Health Sciences \\ Macquarie University, Australia \\ sinantalib@yahoo.co.uk \\ Rupak Kharel \\ School of Engineering \\ Manchester Metropolitan University, UK \\ r.kharel@mmu.ac.uk
}

\begin{abstract}
Internet of Things (IoT) is becoming more and more pervasive in everyday life and connecting an array of diverse physical objects. It is fast growing and receiving a tremendous amount of research focus. Billions of objects communicate each other with or without human intervention to achieve smart applications. Most of the connected devices are constrained nodes to its ecosystem which have limited memories, CPU capabilities and power sources. Therefore, for implementing autonomous smart systems, efficient energy consumption is imperative. This paper introduces a novel scheduling algorithm called Long Hop (LH) first to optimize energy usage on a Wireless Sensor Network (WSN) that enables IoT system. LH algorithm schedules high priority for packets coming with more hops and longer distances to be served first at the cluster head (CH) nodes of the WSN. Since these packets require more links and nodes (thus increased energy and bandwidth usage) to reach the ultimate destination if not prioritized, the proposed algorithm reduces the overall energy usage and minimizes the total number of packets re-transmission and the effective data transmission distances. This improves the overall system performance and elongates the network lifetime.

Index Terms-Internet of Things (IoT), Energy Efficient, Scheduling algorithms, Long Hop First (LH) Algorithm, Wireless Sensor Network (WSN).
\end{abstract}

\section{INTRODUCTION}

Wireless sensor networks (WSNs) are spatially distributed autonomous sensors in a sensing field to monitor specific phenomenons and collect data [1]. WSN is one of the key enabling technologies for the IoT system. IoT is essentially all possible devices connected to the internet that will enable applications to be smart by sensing, harnessing data, making decisions and acting on it, mostly without human interventions [2]. IoT devices are growing at an exponential pace with wearable devices, kitchen appliances, connected cars, healthcare devices, etc becoming more and more commonplace [3].

The growth of devices connectivity will significantly increase over the next few years, according to numbers fore-

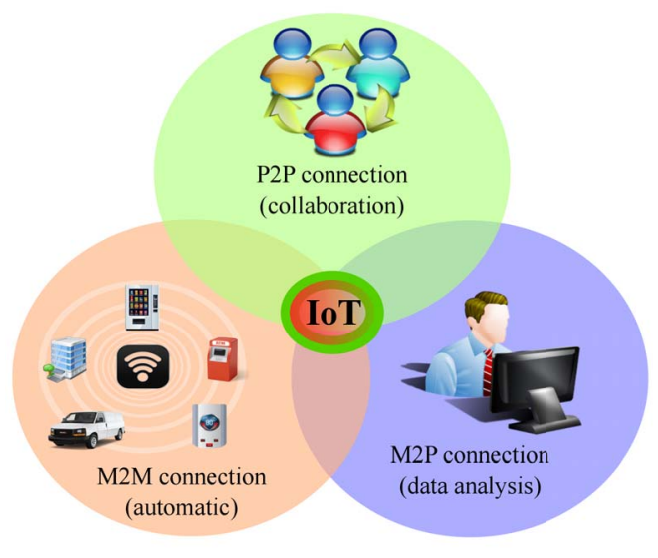

Fig. 1: Internet of things (IoT) elements.

casted by Cisco Systems, from 10 billion in 2014 to 50 billion by 2020 [2]. This will provide a new paradigm of communication between digital contents, computing, applications and services within the physical world. This creates unprecedented opportunities for governments, education, and industries in a wide variety of sectors [4]. The communication of IoT networks combine three categories based on their technology elements that can be seen in Fig. 1.

- People to People (P2P) connection: is the data transfer from one person to the other. It occurs through video call, telephone call, and social communications. It is usually called collaboration connection.

- Machine to People (M2P) connection: is the data transfer from machines such as computing devices, sensor nodes or others to the users for analysis purposes. For 
example: weather forecasting uses smart devices to gather the data from the environment and send it back to the administrators in the control centre for further analysis.

- Machine to Machine (M2M) connection: is the data transfer between devices without human interactions. For instance, a car talking to another car about its speed, lane change or breaking intentions, etc.

The communication of IoT networks combines three main categories based on their technology elements Fig. 1 can be summarized into the simple equation below:

\section{$I o T=$ Human + PhysicalObjects $($ sensors, controllers, devices, storages) + Internet}

Internet of things brings a plethora of devices and infrastructure under the same umbrella. It is a big challenge when more and more smart devices connect to the network. Most of the IoT devices run on batteries or by energy harvesting, which have limited shelf life [5]. This means it will not be rational to waste the energy on the transmission of unneeded data or protocol overheads like existing protocols do such as HTTP and TCP, etc. Therefore, efficient energy consumption is the key issue for longer network and system lifetime. It is related to the sustainability of the network and energy consumption by each single device. The connected devices introduce several services and therefore send heterogeneous packets. These packets access multiple devices and links (mostly wireless communication) to reach the ultimate receiver using multi-hop technology. Multi-hop topology is practically useful for WSN and IoT networks because both techniques are deployed over a wide geographical region [6]. It also provides an opportunity for better quality of services (QoS) and higher network capacity.

During multi hop of packets amongst the nodes especially on a large network, the probability of the packet drops increase. This is because of various factors such as packet arrival rate, timeout for message expiry or simply due to the limitations of node because of its constrained nature (low processing, memory and bandwidth resources). Therefore, to avoid packet loss in the network, receipt acknowledgment of transmitted packets or otherwise retransmission of the lost data packets must happen. Internet control message protocol (ICMP) generated by the network devices is an error-reporting protocol sent to the original source whose IP address is encapsulated in the IP packet. The minimum size of ICMP packet would be 8 bytes of ICMP header +20 bytes of IPv4 or 8 bytes of ICMP header +40 bytes of IPv6 for ICMPv4 and ICMPv6 respectively [7]. Each retransmission will require transmission of these headers and overheads as well. This can significantly impact system performance if the retransmission is not optimized properly. This can more load on the already constrained network and contribute further to the power depletion of the nodes. This affects the quality of service (QoS) and throughput of the network [6] which ultimately drains the node battery leaving the area uncovered, thus reducing the network lifetime.
There are several solutions proposed for reducing energy consumption of WSN and IoT applications. However, most of previous works focused in a small area with less number of nodes which is not consistent with IoT applications. Network of the future will deploy numerous devices covering larger areas. Therefore, the solutions should work on larger areas with higher number of nodes. To this end, the focus and scope of this study is on the energy aspects of the IoT networks. Thus, we propose an energy saving scheme by applying a novel dynamic scheduling algorithm called Long Hop first (LH). LH algorithm operates at the cluster heads (CHs) of a WSN and assigns high priority for messages coming with more hops and from longer distances to be routed first.

This paper is organized as follows: Section II reviews related work from the literature. In Section III, we describe the system model of a IoT system and energy aware-message scheduling algorithm. Performance evaluation and discussion have been done in Section IV. Finally, we conclude our paper in Section $\mathrm{V}$.

\section{RELATED WORK}

The scheduling algorithms are an important aspect for WSN and IoT networks. Several algorithms are proposed for energy consumption optimization and quality of service (QoS) in WSN. Authors in [8] describe the earliest deadline first algorithm (EDF) which is a dynamic scheduling algorithm used for real-time operating systems. EDF requires to sort all tasks based on their deadlines time and gives high priority for packets closest to their exceeded time. However, it does not consider managing time redundancy and packets spend high energy, so scheduling tasks will complete within expire times even in the presence of faults. The proposed work in [9] uses an idea to cluster IoT networks into sub-groups and within each group a message broker was placed. The broker deploys to gather the information from the sensors around it and forward it to the last destination. The short process time (SPT) algorithm implemented at broker level to select and deliver packets first based on their arrival time. Each message is presented as $\operatorname{Mess}\left(R_{\text {time }}, T_{\text {trans }}\right)$, where $R_{\text {time }}$, $T_{\text {trans }}$ are request time and successful transmission time period respectively. It is applied when the network is unstable (traffic intensity $>1$ ). The proposed method promoted IoT system efficiency by improving service response time and reducing the overall energy consumption. Another study [10] schedules packets into three classes priority for large-scale wireless sensor networks. The higher priority was assigned for emergency real time packets and preempt the processing of packets at other queues. Lowest priority packets waited a certain number of time slots after processing higher priority packets. The proposed work improved the system in terms of end-to-end data transmission delay. The study by [11] intends the border nodes of wireless sensor networks. These nodes located between two sensing area consume a large amount of energy due to the listening mode. Thus, S-MAC protocol minimized the listening time of each node. The proposed scheme prolong lifetime and reduce the energy consumption 
for the system. Heterogeneous dual-core processor for IoT applications were carried out by [12]. Authors show that up to 2.62x energy efficiency improvements can be realised without deadline messages expiry. Another approach [13] investigated energy saving scheme by implementing dynamic voltage and frequency scaling (DVFS) scheduling algorithm. DVFS used low time complexity to avoid the deadlines of the real time tasks and showed that it can minimize up to $64 \%$ energy used for each task on a separate core.

Although the previous studies improve on the energy usage, there is no considerable work done on dealing the optimized scheduling for nodes placed in different location (thus still being valid for larger areas and hence scalable). Normally, in multi-hops networks, devices send their packets to the destination through intermediate nodes. In small IoT networks, objects collect the necessary information and then send it to the receiver directly through the $\mathrm{CHs}$ within single or two hops [14]. However, in large networks, the data accesses many links and devices using multi-hop technique to reach the intend destination. Therefore, the data coming from nodes located in longer distances is passed through multiple intermediate nodes to reach the ultimate receiver. Accessing multiple nodes lead to use the bandwidth, throughput and consumption of previous node energy. Therefore, while designing and implementing the efficient energy algorithms, the factor of distance (and hence number of hops) should also be taken into consideration to minimize the power spent and prolong the network lifetime.

In this paper, the proposed scheme is called Long Hop first (LH) scheduling algorithm. It is a scheduling algorithm that focuses on real-time tasks to place processes in a priority queue. LH algorithm assigns high priority for messages coming from far distances and thus accessing multiple nodes to get served first at $\mathrm{CHs}$.

\section{Proposed Energy Efficient Scheduling TECHNIQUE}

In recent study, nodes have been assumed to be deployed in an outdoor area and classified into: i) Sensors and Actuators (SNs) that gather all the necessary information from the environment and send it to $\mathrm{CH}$. ii) Cluster Heads (CHs) receive the heterogeneous data from multiple SNs, compress it, and then send to the intend destination (BS). iii) Base station (BS) is responsible for collecting the information from all sensors through CHs. Our IoT system architecture is clustered into sub-groups, as showed in Fig. 2. Each group has many nodes connected to a single cluster head $(\mathrm{CH})$ node. $\mathrm{CH}$ becomes overloaded due to the number of nodes connected to it. Furthermore, memory of $\mathrm{CHs}$ become full. Therefore, software or hardware flow control must prevent overflow and thus the data loss, otherwise, the node must re-send it again [15].

\section{A. Message Scheduling Algorithm}

Multi-hop technology helps the devices to connect and communicate with each other even if devices are located out of the transmission range. The nodes should depend on some

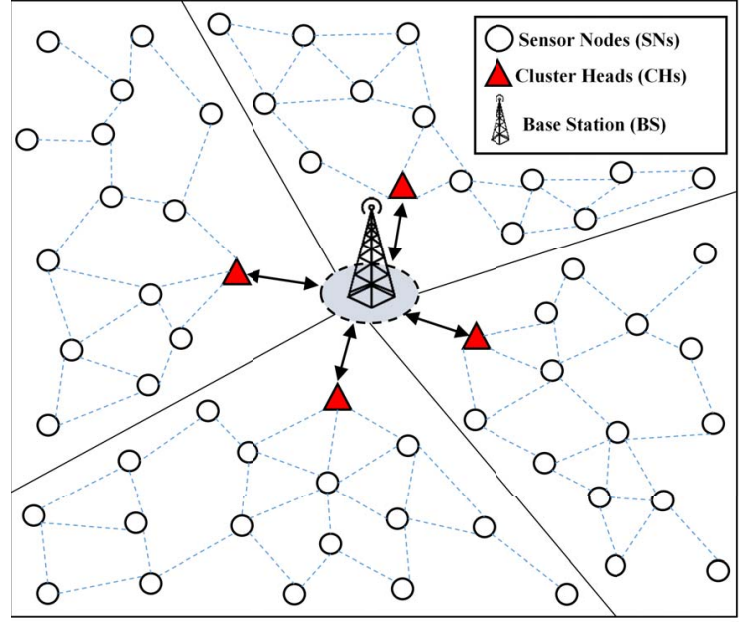

Fig. 2: Typical IoT system architecture.

intermediate nodes for relaying the packets. The packets come from nodes located on the border use high number of hops to reach the ultimate receiver. Thus, these nodes consume larger amount of energy and bandwidth during the transmitting and receiving of the packets by other nodes. Figure 3 shows the measuring energy consumption of tenth nodes deployed randomly in the sensing field. These nodes located in different locations and used some intermediate nodes to deliver their packets. It indicates that packets come from far nodes accessed many devices and used high energy to reach the destination. Due to this reason, it is reasonable to assign high priority for these messages during scheduling algorithm to conserve energy. This is the idea behind the LH algorithm and gives high priority to data based on sensors locations and number of sensors accessed.

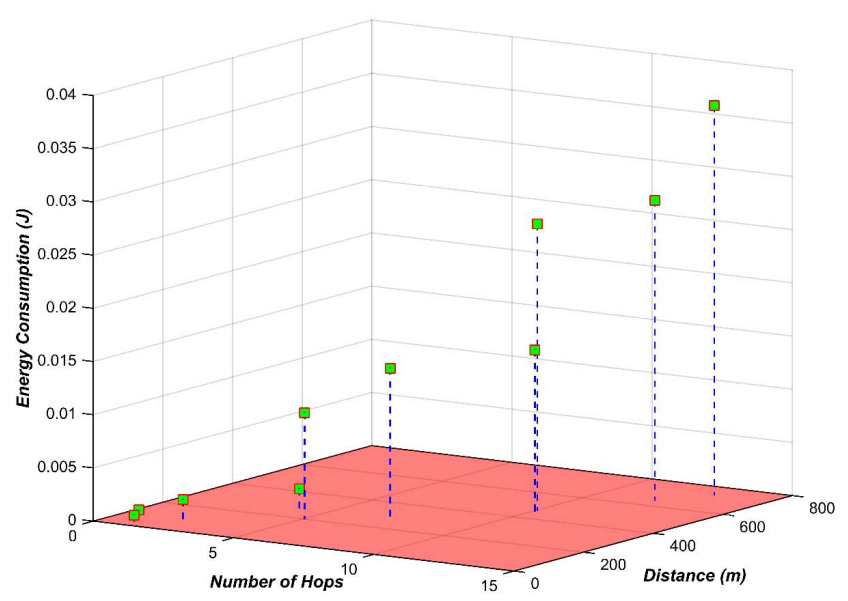

Fig. 3: Energy consumption by number of hops vs distances.

First, we explain how the proposed algorithm works in a cluster of a WSN by analysing the data coming from different nodes to be transmitted to the BS. Figure 4 shows four packets 
at different locations in the cluster making its journey towards the BS. We assume that these packets are transmitted in different time from each sensor and arrive at the same time at $\mathrm{CH}$ where they are placed within a queue at the cluster head $(\mathrm{CH})$ node before delivering to the BS. Some of these packets may be discarded due to quench source, timeout for message expiry, etc. Each task has different number of hops and distances. There must be at least a single task execution through $\mathrm{CH}$ to be forwarded to the exchange centre within one spin. $\mathrm{LH}$ algorithm re-sorts the tasks at $\mathrm{CH}$ based on the biggest number of hops and longer distances to forward it first to ultimate receiver. If there are two packets with equal number of hops (as with orange and yellow colour in Fig. 4), the algorithm takes the second parameter (longer distance) into consideration. Therefore, orange packet gets delivered first at $\mathrm{CH}$ due to its sensor location. $M / M / 1$ queuing model has been used to calculate service rate and arrival rate for all messages coming from devices. $M / M / 1$ is a queuing theory within the mathematical theory of probability that shows the queue length of a single server in the system. Service times have an exponential distribution and exponential arrivals are determined by a Poisson process [16]. The service rate and arrival rate for $m$ messages are introduced by $\mu$ and $\lambda$ respectively. After evaluating the system traffic at the cluster heads, traffic intensity $(P)$ should be less than 1 . However, if $P$ is larger than 1 , the proposed algorithm avoids packets accessing many devices and links from retransmission. Figure 5 explains the algorithm with the aid of a flowchart.

$$
\begin{gathered}
\lambda=\frac{1}{R_{\text {time }}} \\
\mu=\frac{1}{T_{\text {trans }}} \\
P=\frac{\lambda}{\mu}
\end{gathered}
$$

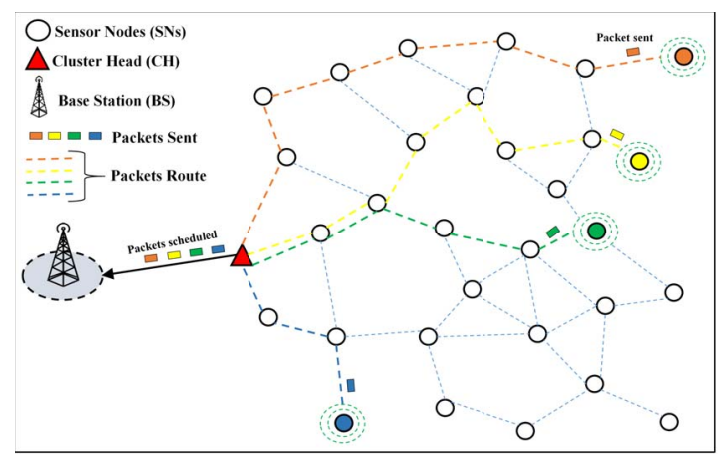

Fig. 4: The partial schedule of four tasks under LH algorithm.

\section{B. Energy Consumption Model}

The aim of this study is to implement message scheduling algorithm that minimizes energy consumption and elongates lifetime of the network. Most of the energy is consumed in listening, transmitting and receiving of packets in the

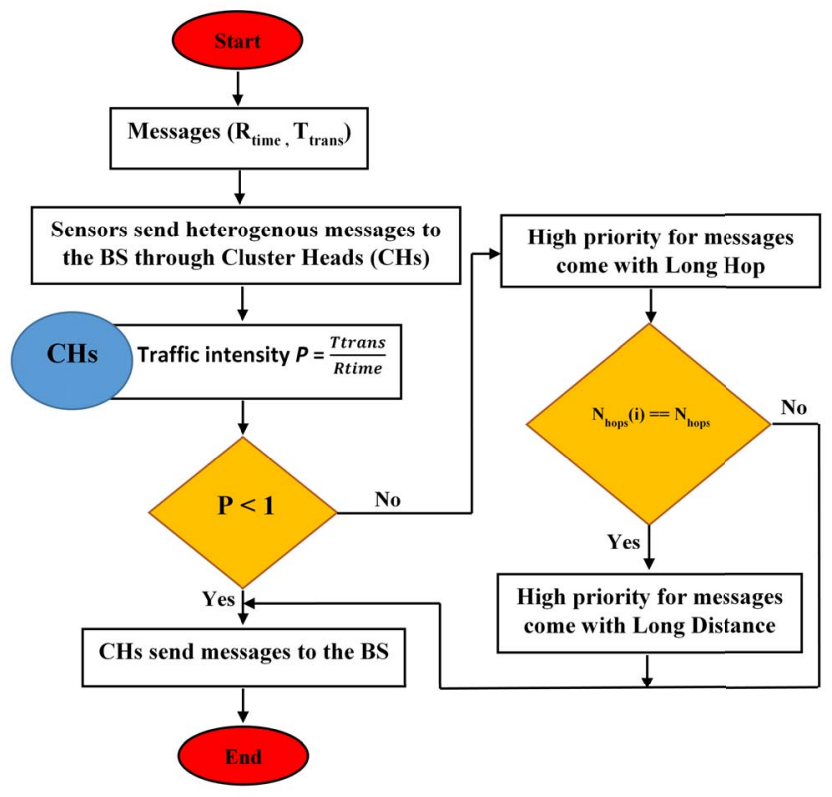

Fig. 5: Flow-chart of LH procedure.

network. A common power model [17] is used and shown in Fig. 6. Sensors follow shortest path route to deliver their packets to the next hop. Dijkstra algorithm [18] [19] has been applied to find the shortest path between nodes. The total energy [9] spent by the system can be represented as follows:

- To transmit a number of bits:

$$
E_{T x}=k\left(E_{\text {elec }}+\epsilon_{a m p} * d^{2}\right)
$$

- To receive a number of bits:

$$
E_{R x}=\left(k * E_{\text {elec }}\right)
$$

- Total energy used by each sensor:

$$
E_{\text {Total }}=L\left(E_{T x}\right)+M\left(E_{R x}\right)
$$

where $E_{T x}$ is the energy dissipated to disseminate number of bits $(k)$ from the source to the next object. $E_{R x}$ is the energy depleted to receive chunk of bits. $E_{\text {elec }}$ is presented the energy depleted to run the receiver or transmitter circuitry, and $\epsilon_{a m p}$ is the energy dissipated in transmission to amplify the signal enough to reach the next target. $L$ and $M$ are number of transmitted and received packets from a sensor node respectively. The distance between two transmitters is denoted as $d$.

\section{Performance Evaluation and Discussion}

In this section, we evaluate and analyse the performance of the proposed scheme by using Matlab simulation. IoT consists of number of devices/things deploy randomly in a large scale. Therefore, the simulation is performed with 100 nodes that are distributed randomly in an area of $(500 \mathrm{x}$ 500) $\mathrm{m}^{2}$, using mesh topology consisting multi-hop network scenario. We also assume that the sensor nodes are deployed 


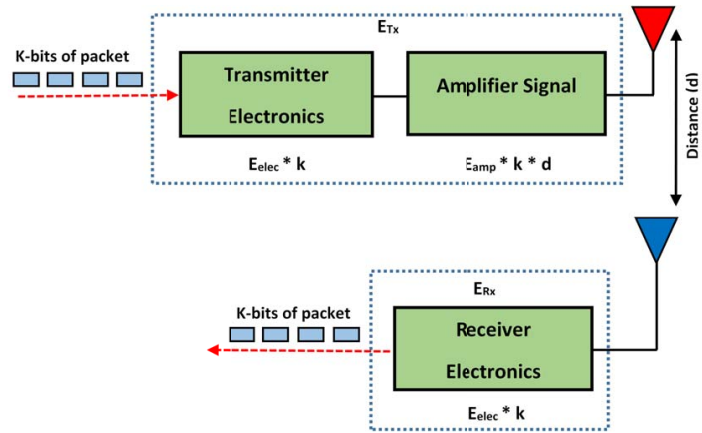

Fig. 6: The wireless communication power model.

outdoor to monitor the environment that send the information to the central application through $\mathrm{CH}$ nodes. $\mathrm{CHs}$ job is to collect the data coming from the sensors, process and deliver it to the BS. In every round, each sensor sends 250 bytes to the $\mathrm{BS}$ through the CHs. The BS is set at the centre of the square field. The assumptions and parameters used in the simulation model are shown in the table I.

TABLE I: Parameters used in the simulation

\begin{tabular}{||cc||}
\hline Parameter & Value \\
\hline Electronics Energy $\left(E_{\text {elec }}\right)$ & $50 \mathrm{~nJ} / \mathrm{bit}$ \\
Initial energy of node $\left(E_{\text {init }}\right)$ & $0.25 \mathrm{~J}$ \\
Communication energy $\left(\epsilon_{m p}\right)$ & $10 \mathrm{pJ} / \mathrm{bit} / \mathrm{m}^{2}$ \\
Threshold value of distance $\left(d_{0}\right)$ & $87 \mathrm{~m}$ \\
Buffer size & $202 \mathrm{bytes}$ \\
Payload size & 210 bytes \\
Header size & 40 bytes \\
Retransmission overhead size & 8 bytes + header size \\
Number of nodes $(N)$ & 100 \\
Sensing Area & $500 \mathrm{~m} \times 500 \mathrm{~m}$ \\
\hline
\end{tabular}

A performance comparison is done when the proposed scheduling algorithm and no scheduling is used. It is important to minimize the retransmission packets that can further reduce the processing time, capacity used, and energy consumption of the network. Figure 7 shows that the proposed algorithm has lower number of transmitting and receiving packets in the network. This means that the use of LH algorithm has led to the reduction in total transmitting and receiving power thus and ultimately extending the network lifetime.

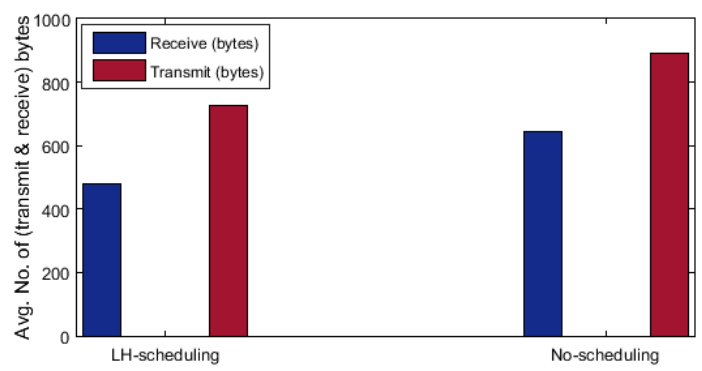

Fig. 7: Avg. number of (transmitting and receiving) packets.

Number of hops is the summation of data relays on multihop communication to reach the intended destination. A hop occurs when the packet is passed to the next network device.

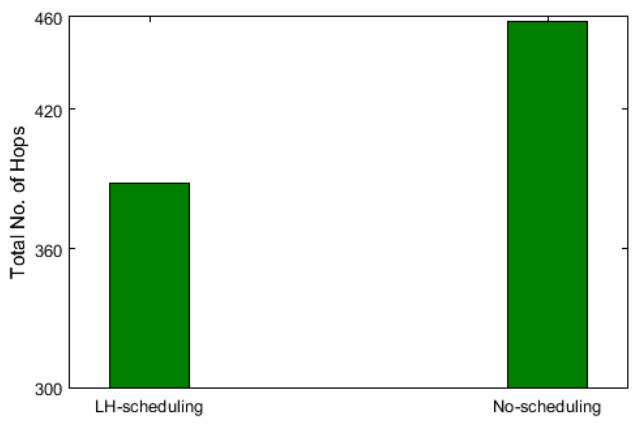

Fig. 8: Total Number of Hops.

Next hop is a routing term that refers to the next device based on the type of routing algorithm is used. The purpose is to minimize the power by reducing the relay packets from the sender to the receiver. Figure 8 shows that proposed algorithm has minimum number of hops compared to when the algorithm is not implemented.

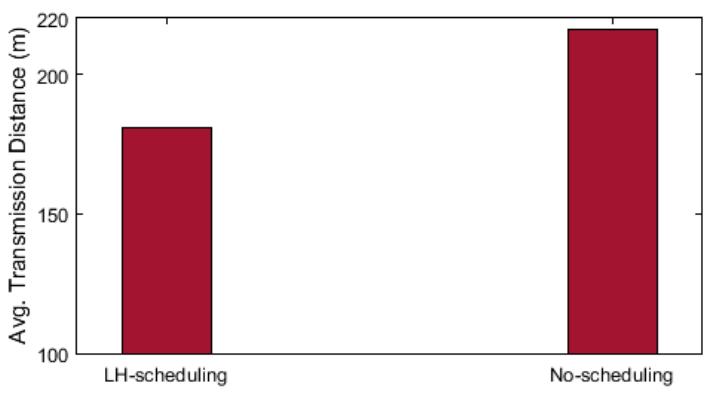

Fig. 9: Avg. Transmission Distances (m).

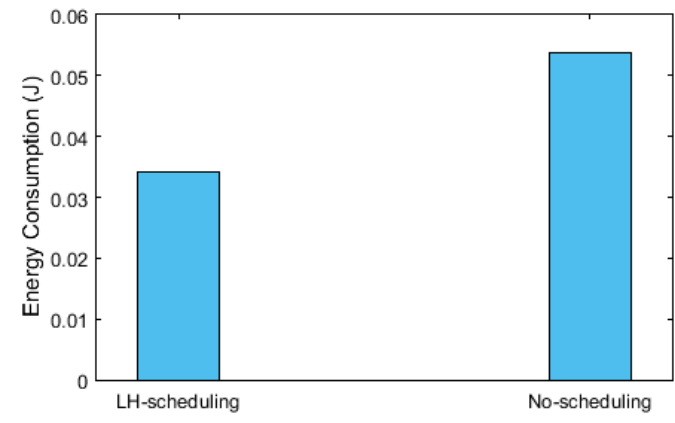

Fig. 10: Avg. Energy Consumption.

Transmission distance is the physical path (wire/wireless) between transmitter and receiver within single hop or multihop communication. It is only plausible to say that longer distances from sources to the intended destination will consume more power. Therefore, reducing the average transmission distance for the packets will have positive impact on the energy consumption and delay time. Figure 9 depicts the average transmission distance from each single node to the BS 
showing that when the algorithm is implemented, the average transmission distance is reduced.

Figure 10 shows the average energy consumption for each round. According to the Figs. [7 - 9], the proposed algorithm reveals less number of hops and transmission distances. As a result, the energy consumption of the proposed scheme is lower.

\section{CONCLUSION}

This paper proposed an energy efficient messaging scheduling algorithm for IoT applications to be implemented at the $\mathrm{CH}$ node of the WSN. Since packet loss occurs at $\mathrm{CH}$ nodes due to quench, time to live exceeding for packets, or destination being unreachable, application of the proposed Long Hop first (LH) algorithm on the $\mathrm{CH}$ nodes shows promising results for energy optimization. The proposed algorithm assigns high priority for messages coming from far distances to be routed first thus preventing re-transmission. The preliminary results showed the effectiveness and the efficiency of the proposed algorithm to reduce the number of re-transmission packets, energy consumption, transmission distance, number of hops, and thus elongate the network lifetime. The future work is to apply the proposed scheme into a larger area with increased number of nodes to show the scalability and compare the performance with existing energy efficient methods.

\section{ACKNOWLEDGMENT}

The authors would like to thank Ministry of Higher Education and Scientific Research (Iraq) and University of Diyala for the funding to conduct the research. Also, many thanks to Manchester Metropolitan University for their support.

\section{REFERENCES}

[1] M. Hammoudeh, F. Al-fayez, H. Lloyd, R. Newman, and B. Adebisi, "System : Deployment Issues and Routing Protocols," IEEE SENSORS, vol. 17 , no. 8, pp. 2572-2582, 2017.

[2] W. Stallings, Foundations of Modern Networking: SDN, NFV, QoE, IoT, and Cloud. Addison-Wesley Professional, 2015.

[3] H. Ghayvat, S. Mukhopadhyay, X. Gui, and N. Suryadevara, "Wsn-and iot-based smart homes and their extension to smart buildings," Sensors, vol. 15, no. 5, pp. 10350-10379, 2015.

[4] D. R. Dandekar and P. Deshmukh, "Energy balancing multiple sink optimal deployment in multi-hop wireless sensor networks," in Advance Computing Conference (IACC), 2013 IEEE 3rd International. IEEE, 2013.

[5] M. Hammoudeh and R. Newman, "Adaptive routing in wireless sensor networks : QoS optimisation for enhanced application performance," Information Fusion, vol. 22, pp. 3-15, 2015.

[6] A. Abuarqoub, M. Hammoudeh, B. Adebisi, S. Jabbar, A. Bounceur, and H. Al-Bashar, "Dynamic clustering and management of mobile wireless sensor networks," Comput. Netw., vol. 117, no. C, pp. 62-75, Apr 2017.

[7] S. Hagen, "IPV6 Essentials," Grundlagen - Funktionalität, Integration. 2nd edn. Sunny Edition, 2009.

[8] H. Wang, Jie Jin, Zhijun Wang, and L. Shu, "On a novel property of the earliest deadline first algorithm," 2011.

[9] S. Abdullah and K. Yang, "An energy-efficient message scheduling algorithm in Internet of Things environment," in 9th International Wireless Communications and Mobile Computing Conference (IWCMC). IEEE, jul 2013.

[10] L. Karim, N. Nasser, T. Taleb, and A. Alqallaf, "An Efficient Priority Packet Scheduling Algorithm for Wireless Sensor Network," Communications (ICC), 2012 IEEE International Conference, pp. 334-338, 2012.
[11] D. Saha, M. Yousuf, and M. Matin, "Energy efficient scheduling algorithm for s-mac protocol in wireless sensor network," International Journal of Wireless \& Mobile Networks, vol. 3, no. 6, p. 129, 2011.

[12] Z. Wang, Y. Liu, Y. Sun, Y. Li, D. Zhang, and H. Yang, "An energyefficient heterogeneous dual-core processor for Internet of Things," 2015 IEEE International Symposium on Circuits and Systems (ISCAS), May 2015.

[13] W. Y. Lee, "Energy-Saving DVFS Scheduling of Multiple Periodic Real-Time Tasks on Multi-core Processors," in 2009 13th IEEE/ACM International Symposium on Distributed Simulation and Real Time Applications. IEEE, 2009.

[14] Z. Vincze, R. Vida, and A. Vidacs, "Deploying Multiple Sinks in Multihop Wireless Sensor Networks," in IEEE International Conference on Pervasive Services. IEEE, jul 2007.

[15] XBee Modules, "XBee ® /XBee-PRO ® RF Modules," in Product Manual v1. xEx-802.15.4 Protocol, 2009.

[16] S. Abdullah and K. Yang, "An Energy Efficient Message Scheduling Algorithm Considering Node Failure in IoT Environment," Wireless Personal Communications, vol. 79, no. 3, pp. 1815-1835, Dec 2014.

[17] J.-Y. Chang and T.-H. Shen, "An Efficient Tree-Based Power Saving Scheme for Wireless Sensor Networks With Mobile Sink," IEEE Sensors Journal, vol. 16, no. 20, pp. 7545-7557, oct 2016.

[18] S. Broumi, A. Bakal, M. Talea, F. Smarandache, and L. Vladareanu, "Applying dijkstra algorithm for solving neutrosophic shortest path problem," in 2016 International Conference on Advanced Mechatronic Systems (ICAMechS), Nov 2016.

[19] A. M. Ahmed, S. H. Ahmed, and O. H. Ahmed, "Dijkstra algorithm applied: Design and implementation of a framework to find nearest hotels and booking systems in iraqi," in 2017 International Conference on Current Research in Computer Science and Information Technology (ICCIT), April 2017. 